\title{
Research
}

\section{Clinical predictors of antibiotic prescribing for acutely ill children in primary care:}

\author{
an observational study
}

\begin{abstract}
Background

Antibiotic overuse and inappropriate prescribing drive antibiotic resistance. Children account for a high proportion of antibiotics prescribed in primary care.

\section{Aim}

To determine the predictors of antibiotic prescription in young children presenting to UK general practices with acute illness.
\end{abstract}

\section{Design and setting}

Prospective observational study in general practices in Wales.

\section{Method}

A total of 999 children were recruited from 13

practices between March 2008 and July 2010.

Multilevel, multivariable logistic regression analysis was performed to determine predictors of antibiotic prescribing

\section{Results}

Oral antibiotics were prescribed to 261 children (26.1\%). Respiratory infections were responsible for $77.4 \%$ of antibiotic prescriptions. The multivariable model included 719 children. Children were more likely to be prescribed antibiotics if they were older lodds ratio [OR] $1.3 ; 95 \%$ confidence intervals $[\mathrm{Cl}]=1.1$ to 1.7 ); presented with poor sleep (OR 2.7: $95 \% \mathrm{Cl}=1.5$ to 5.0); had abnormal ear (OR $6.5 ; 95 \% \mathrm{Cl}=2.5$ to 17.2), throat (OR 2.2; $95 \% \mathrm{Cl}=1.1$ to 4.5 ) or chest examination (OR 13.6; $95 \% \mathrm{Cl}=5.8$ to 32.2): were diagnosed with lower respiratory tract infection (OR 9.5; $95 \% \mathrm{Cl}=3.7$ to 25.5 ), tonsillitis/ sore throat (OR 119.3; $95 \% \mathrm{Cl}=28.2$ to 504.6), ear infection (OR 26.5; 95\% $\mathrm{Cl}=7.4$ to 95.7 ) or urinary tract infection (OR $12.7 ; 95 \% \mathrm{Cl}=4.4$ to 36.5): or if the responsible clinician perceived the child to be moderately to severely unwell (OR 4.0 $95 \% \mathrm{Cl}=1.4$ to 11.4 . . The area under the receiver operating characteristic curve was 0.9371 .

\section{Conclusion}

Respiratory infections were responsible for $74.4 \%$ of antibiotic prescriptions. Diagnoses of tonsillitis, sore throat, or ear infection were associated most with antibiotic prescribing. Diagnosis seemed to be more important than abnormal examination findings in predicting antibiotic prescribing although these were correlated.

\section{Keywords}

acute disease; antibacterial agents; antibiotic prescribing; children; infection; primary care.

\section{BACKGROUND}

Antibiotic resistance is an increasing public health problem worldwide, and has been highlighted as a national and international priority ${ }^{1,2}$ Antibiotic prescribing drives antibiotic resistance on both a population and individual level. ${ }^{1,3-7}$ In addition, antibiotics may cause adverse effects and their overuse can result in 'medicalisation' of self-limiting illness. ${ }^{8,9}$

In the UK, primary care is responsible for $80 \%$ of (human) antibiotic prescriptions, and the highest prescription rate is in children aged 1-4 years. ${ }^{10-12}$ Both the decision to prescribe and choice of antibiotic are complex, and influenced by both clinical and non-clinical factors. ${ }^{13-20}$ Most studies have focused on prescribing for respiratory tract infections (RTIs). ${ }^{17,18,21}$ Some studies have considered antibiotic prescribing more generally in children but there are few studies based in UK general practice. ${ }^{16,20}$

The UK National Institute for Health and Care Excellence (NICE) guidelines recommend 'no' or 'delayed' antibiotic prescribing for most RTIs. ${ }^{22}$ Antibiotics are not beneficial for most upper respiratory tract infections (URTIs) including tonsillitis, otitis, acute cough, and colds, with little impact on illness course or complication incidence.22,23 Antibiotic prescribing for specific RTI diagnoses in children decreased in the late 1990s but has been increasing

K O'Brien, PhD, MRCGP, clinical lecturer, Division of Population Medicine; A Edwards, PhD, MRCGP director, Division of Population Medicine and professor of primary care, School of Medicine; M Kelson, PhD, senior statistician; K Hood, PhD, director of clinical trials and professor of statistics, South East Wales Trials Unit, Cardiff University, Cardiff. TW Bellis, BSc, MBBCh, FY1 doctor, Great Western Hospital, Swindon. CC Butler, PhD, FRCGP. professor of primary care, Nuffield Department of Primary Care Health Sciences Oxford University, Oxford and Division of Population Medicine, School of Medicine, Cardiff University, Cardiff, UK. again since the early 2000s, especially for non-specific URTI and 'abnormal symptoms and signs. ${ }^{12,16,24}$

There is wide variation in antibiotic prescribing between countries, general practices, and individual doctors. $8,11,12,20,25$ Patient factors, such as deprivation and comorbidities; clinician factors, such as previous experience, training, and attitudes towards antibiotics; the doctorpatient relationship and perceived patient expectation; and other factors such as time and workload pressures, have been identified as influencing prescribing. 15,19,26 Diagnostic uncertainty and concern about serious illness and possible complications may also be contributory factors. ${ }^{19,27}$

Presenting symptoms and signs are considered by clinicians as important in deciding whether to prescribe antibiotics or not. ${ }^{19}$ However, it is unclear which clinical or non-clinical features are most likely to result in antibiotic prescribing. Several studies, including one UK-based study, have examined antibiotic prescribing in primary care using routinely collected data. ${ }^{20,25,28,29}$ Analyses of large routinely collected data are extremely important in understanding the scale of antibiotic prescribing and these studies can also provide information on the type of antibiotic and condition it is prescribed for. However, these studies are less able to examine in detail the clinical and

\section{Address for correspondence}

Kathryn O'Brien, Cardiff University, School of Medicine, Division of Population Medicine, Neuadd Meirionnydd, Heath Park, Cardiff CF14 4YS, UK.

E-mail: obrienkadcardiff.ac.uk

Submitted: 16 December 2014; Editor's response: 25 February 2015; final acceptance: 12 May 2015 ()British Journal of General Practice

This is the full-length article (published online 1 Sep 2015) of an abridged version published in print. Cite this version as: Br J Gen Pract 2015; DOI: 10.3399/bjgp15X686497 


\section{How this fits in}

Trials and systematic reviews have found evidence for limited or no benefit for antibiotics in most childhood common infections. The National Institute for Health and Care Excellence guidelines recommend against the routine use of antibiotics for common childhood respiratory tract infections. There is limited evidence from prospective studies of antibiotic prescribing rates for children with unselected illness in primary care. Respiratory infections are still responsible for more than $70 \%$ of antibiotic prescriptions in children despite evidence-based guidelines that most such prescriptions are unwarranted. Diagnoses of tonsillitis, sore throat, or ear infection were most associated with antibiotic prescribing with antibiotics.

non-clinical features that are most predictive of antibiotic prescribing at the individual level. For this, prospective observational studies are needed.

A study in the Netherlands described symptoms and signs associated with antibiotic prescribing in 443 febrile children. ${ }^{16}$ The study found that parental concern, ill appearance, altered sleep patterns due to earache, signs of throat infection, and reduced urine production were all associated with antibiotic prescribing A Danish study of 954 children with otitis media found fever and a red tympanic membrane were associated with antibiotic prescribing. ${ }^{18}$ The study in the Netherlands only included children with fever presenting to an out-of-hours service and the Danish study only included children with otitis media. ${ }^{16,18}$ The Netherlands and Denmark are also among the countries with the lowest antibiotic prescribing rates in Europe and the US. ${ }^{5}$ No prospective studies were found that described factors associated with antibiotic prescribing generally in ill children presenting routinely in UK primary care.

The aim of this study was to describe antibiotic prescribing in consecutive young children $(<5$ years) presenting to UK general practice with an acute illness (<28 days duration) and to identify which clinical features were most associated with antibiotic prescribing.

\section{METHOD}

\section{Inclusion criteria}

A cohort of consecutively presenting acutely ill children was recruited from general practices in Wales between March 2008 and July 2010. The cohort was originally

recruited to determine childhood UTI prevalence among unselected acutely ill children. ${ }^{30}$ Following a pilot study of feasibility, practices in South Wales lall practices in the Cardiff and Vale areal were sent a letter inviting them to take part in the study. ${ }^{31}$ Subsequently, recruitment was extended and 13 practices across Wales agreed to participate in the study. Children were eligible for recruitment if they were aged $<5$ years and presented with an acute illness of $<28$ days duration. Children were excluded if they were currently receiving immunosuppressive treatment Ichemotherapy or oral/intramuscular steroids $\geq 2$ weeks), long-term antibiotic treatment ( $>28$ days), or had already participated in the study.

\section{Data collection}

Demographic data and clinical data were recorded by a practice or research nurse. Townsend scores for the general practices were calculated as an estimate for deprivation. 32,33 Clinical data included presenting symptoms and previous medical history; and basic observations included temperature, pulse, and respiratory rates. Symptoms were recorded by ticking a list provided on the case record form. Examination findings, working diagnosis, management, and whether oral antibiotics were prescribed were recorded by the treating clinician. Parents and clinicians were both asked to give an overall impression of the child's illness severity on a 5-point scale from 0 (not unwell) to 4 (severely unwell).

\section{Data analysis}

Data were analysed using SPSS (version 18.0) and $R$ (version 3.0.2). For symptoms, if the item was not ticked as 'present", it was assumed that it was not present and analysed together with those ticked as 'absent'. For examination findings and working diagnosis, missing information was analysed as 'not examined' and 'no diagnosis given', respectively. For the analysis, the clinicians' working diagnoses were further grouped: 'pneumonia', 'chest infection', 'bronchitis', 'bronchiolitis', and 'croup' were grouped with lower respiratory tract infection (LRTI); 34 'throat infection' and 'sore throat' were grouped with 'tonsillitis'; 'coryza' and 'cough' were grouped with URTI; exacerbation of asthma was included in the 'other' category unless URTI or LRTI was also mentioned. For the parent/carer and clinician illness severity measures, scores of 2, 3, and 4 (moderately, very, or severely unwell) were grouped together due to low numbers in these groups compared 


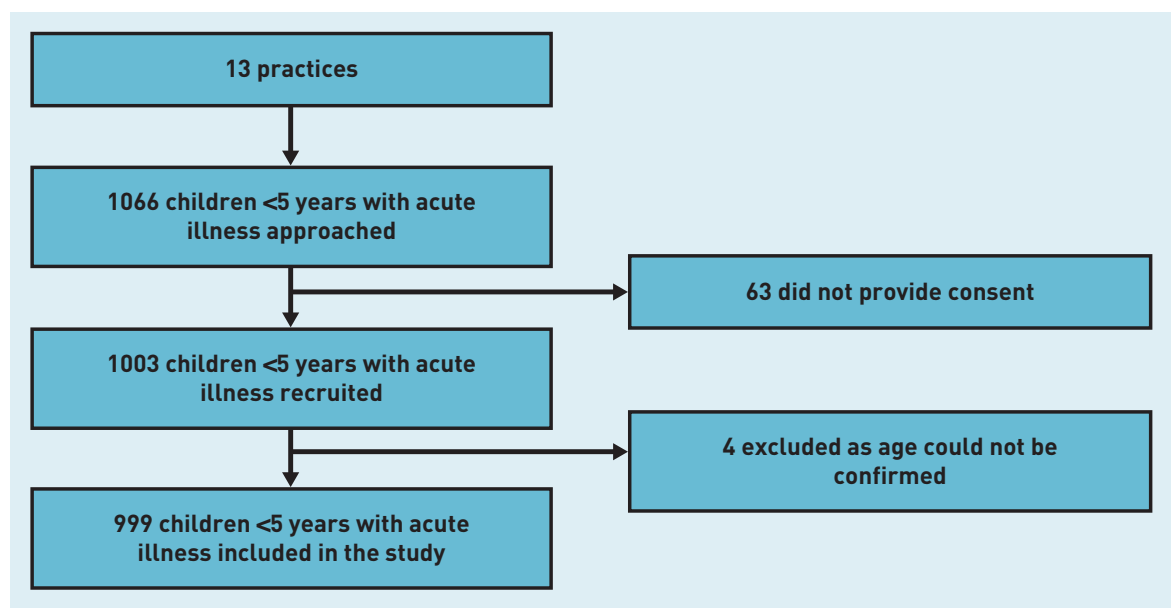

Figure 1. Flow diagram of patient recruitment. with normal/not unwell (score of 0 ) and slightly unwell (score of 1).

Tachycardia and tachypnoea were calculated using the heart rates, respiratory rates, and ages of the children according to the categories given in the NICE guideline for feverish illness. ${ }^{35}$

Multilevel modelling was used to account for clustering at the level of the general practices. Multilevel logistic regression was carried out using $R$ (version 3.0.2). All clinical factors with a $P$-value $<0.1$ on crude (unadjusted) analyses were entered into a multivariable (adjusted) analysis, where missing data were $<30 \%$. Variables with missing data of $\geq 30 \%$ and variables with a low overall prevalence, of $<10 \%$, were excluded from the multilevel model, due to insufficient data. To estimate the amount of influence the clusters (13 practices) had on the overall variation in antibiotic prescribing. the intra-class correlation coefficient (ICC) was calculated using the $\frac{\pi^{2}}{3}$ estimator. ${ }^{36}$

A sensitivity analysis was conducted to investigate the impact of missing data. Multiple imputation with chained equations was implemented producing five imputed datasets. ${ }^{37}$ Each fixed effect variable in the final model was imputed using all other fixed effect variables. The final model was fitted to these five and the results pooled.

\section{RESULTS}

Thirteen practices in Wales took part in the study. Practices were represented in affluent and less affluent areas covering Townsend scores from all quintiles. Practice list size ranged from 2121 to 25251 . Numbers of children recruited into the cohort from each practice ranged from 14 to 251 . Overall, 1003 children were recruited. Four children were later excluded because their date of birth was incomplete or incorrectly recorded, and their age could not be confirmed (Figure 1). Therefore, 999 children were included in this study. Nine practices completed recruitment logs and identified 63 children who were eligible but who were not recruited. Reasons for non-recruitment included consent not given; parent/carer did not have time; or the parent felt that the child was too ill to participate.

Of the 999 children included in this study, 498 (49.8\%) were female and the median age was 1.9 years (interquartile range $[\mathrm{IQR}]=0.9-$ 3.3). The median age of non-recruited children was 1.6 years $(I Q R=1.0-3.1)$, and 34 (53.9\%) were male. RTIs were responsible for 496 (49.6\%) consultations. The most frequent diagnosis was URTI in 297 (29.7\%) children (Table 1). Oral antibiotics were prescribed in $261(26.1 \%)$ children (Table 1). Antibiotics were frequently prescribed for children with a working diagnosis of tonsillitis or sore throat $(54 / 58,93.1 \%)$, ear infections (42/51. 82.4\%), and LRTI (63/90, 70.0\%). LRTI was responsible for $63 / 261(24.1 \%)$ of all antibiotic prescriptions, and RTIs more widely were responsible for $202(77.4 \%)$ of all antibiotic prescriptions (Table 1).

The mean age of children prescribed antibiotics was 2.52 years (standard deviation $[S D]=1.30$ ) and the mean age of children not prescribed antibiotics was 1.99 years $(S D=1.42)$. Amoxicillin was the most commonly prescribed antibiotic, in 150 (57.5\%) antibiotic prescriptions. Penicillin was prescribed in 38 (14.6\%); erythromycin in $20(7.7 \%)$; trimethoprim in 19 (7.3\%); cefalexin in 11 (4.2\%); co-amoxiclav in 10 (3.8\%); flucloxacillin in $10(3.8 \%)$; clarithromycin in $2(0.8 \%)$; and metronidazole in 1 (0.4\%).

Although requested for all participants, examination recordings were incomplete for temperature (recorded in 757; 75.8\%), pulse 
Table 2. Variables associated with antibiotic prescribing at $P<0.01$ that were excluded from the multilevel logistic regression model due to low overall prevalence

\begin{tabular}{|c|c|c|c|c|}
\hline Variable & $\begin{array}{c}\text { Children affected, } \\
\qquad n(\%)\end{array}$ & $\begin{array}{c}\text { Prescribed } \\
\text { antibiotics, } n(\%)\end{array}$ & Odds ratio & $P$-value \\
\hline Muscle aches/pains & 90 (9.0) & $37(41.1)$ & 2.14 & 0.001 \\
\hline Colic/grimacing/pulling up legs & $46(4.6)$ & $7(15.2)$ & 0.49 & 0.085 \\
\hline Haematuria & $4(0.4)$ & $4(100)$ & $N / A$ & $0.005^{a}$ \\
\hline Poor urine flow & 37 (3.7) & 17 (45.9) & 2.50 & 0.005 \\
\hline Past history of UTI & $58(5.8)$ & 22 (37.9) & 1.80 & 0.035 \\
\hline Past history of hypertension & $2(0.2)$ & $2(100)$ & & 0.017 \\
\hline Same day hospital admission & $20(2.0)$ & 0 & & 0.007 \\
\hline
\end{tabular}

(recorded in 502; 50.3\%), and respiratory rate (recorded in 454; 45.4\%). Pulse and respiratory rate were analysed as binary variables: tachycardia (high pulse for age) or normal pulse rate for age; and tachypnoea (high respiratory rate for age) or normal respiratory rate for age. Only $2.4 \%$ of children were classified as tachycardic. There was no association between tachycardia and antibiotic prescribing, with $4 / 140(2.9 \%)$ of those prescribed antibiotics classified as tachycardic, and 8/362 (2.2\%) of those not prescribed antibiotics classified as tachycardic $(P=0.75)$. Tachypnoea was present in $13.9 \%$ of children. Among those prescribed antibiotics, 12/129 (9.3\%) were classified as tachypnoeic and 51/325 (15.7\%) of those not prescribed antibiotics were classified as tachypnoeic ( $P=0.08$ ).

Parent/carer-rated illness severity scores were provided for 931 children (93.2\%). Children were most frequently scored as 1 (slightly unwell) in 374 (40.2\%). Clinician impression was completed for 946 children (94.7\%). Most children, 519 (54.9\%), were considered to be 'slightly unwell'. Antibiotics were prescribed in 103/198 (52.0\%) children scored as 2, 3, or 4 (moderately, very, or severely unwell) by clinicians, in 136/519 (26.2\%) scored as 1 (slightly unwell), and in $18 / 229(7.9 \%)$ scored as 0 (normal/not unwell).

\section{Multilevel multivariable analysis}

The ICC for clustering by practice was $9.1 \%$. This suggests that approximately $9.1 \%$ of the overall variation in prescribing outcomes is attributable to practices rather than patients.

Variables that were significant in the crude (unadjusted) analysis but excluded from the multivariable (adjusted) model due to a low overall prevalence (of $<10 \%$ ) were: muscle aches/pains, colic, haematuria, poor urine flow, past history of UTI, past history of hypertension, and same day hospital referral (Table 2). Respiratory rate (tachypnoea) was excluded from the multivariable (adjusted) model due to missing data in 55\% (respiratory rate recorded in only 454/999).

Multiple imputation. There were missing values for three of the variables included in the final model: whether the child was categorised as not unwell; mildly unwell; or moderately, very, or severely unwell by the parent/carer (68 missing observations); whether the child was categorised as not unwell; mildly unwell; or moderately, very, or severely unwell by the clinician (53 missing observations); and temperature measured at the time of presentation $(242$ missing observations). Multiple imputation did not appreciably alter the conclusions drawn from the complete case analysis (results presented in Table 3). The ICCs for the imputed models were lower than observed in the complete case analysis (mean ICC: $7.0 \%$, range $6.9-7.2 \%$ ).

The results for the multivariable (adjusted) logistic regression analysis on complete cases is shown in Table 3 alongside the multivariable (adjusted) logistic regression analysis with multiple imputation. The results of the complete case analysis will be described throughout the rest of the results.

Children were more likely to be prescribed antibiotics if they were older. For each 1-year increase in age, the odds of being prescribed an antibiotic increased by $33 \%$ (OR $1.3 ; 95 \% \mathrm{Cl}=1.1$ to 1.7 ). The only symptom found to increase the likelihood of an antibiotic prescription in the logistic regression model was poorer sleep, with those children twice as likely to receive antibiotics (OR 2.7; $95 \% \mathrm{Cl}=1.5$ to 5.0 ). Difficulty breathing was found to reduce the likelihood of antibiotics in the model IOR $0.4 ; 95 \% \mathrm{Cl}=0.2$ to 0.8 ). Antibiotics were more likely to be prescribed if there were abnormal findings on ear leardrum or ear canal red or inflamed; or discharge or blood seen; OR 6.5; $95 \% \mathrm{Cl}=2.5$ to 17.2 ), throat (red/inflamed or swollen tonsils or discharge/pus; OR 2.2; $95 \% \mathrm{Cl}=1.1$ to 4.5 ), or chest examination lwheeze or crackles or use of accessory muscles or recession; OR $13.6 ; 95 \% \mathrm{Cl}=5.8$ to 32.2 ).

When GP working diagnosis was considered, URTI was the most prevalent diagnosis and was used as the reference category for comparison with other working diagnoses. Antibiotics were more likely to be prescribed when GPs gave a 
Table 3. Multilevel logistic regression: complete case analysis and analysis with multiple imputation showing all the variables entered into the model and association with antibiotic prescription

\begin{tabular}{|c|c|c|c|c|c|c|}
\hline \multirow[b]{2}{*}{ Variable } & \multicolumn{3}{|c|}{ Complete case } & \multicolumn{3}{|c|}{ Multiple imputation } \\
\hline & Odds ratio & $95 \% \mathrm{Cl}$ & $P$-value & Odds ratio & $95 \% \mathrm{Cl}$ & $P$-value \\
\hline \multicolumn{7}{|l|}{ Signs, symptoms, and past history } \\
\hline Runny or blocked nose & 1.7 & 0.8 to 3.4 & 0.160 & 1.5 & 0.9 to 2.8 & 0.140 \\
\hline Sore throat & 1.1 & 0.6 to 1.9 & 0.850 & 1.1 & 0.7 to 1.9 & 0.610 \\
\hline Earache or holding ear & 0.5 & 0.3 to 1.0 & 0.040 & 0.7 & 0.4 to 1.1 & 0.140 \\
\hline Cough & 0.7 & 0.3 to 1.4 & 0.280 & 0.8 & 0.5 to 1.5 & 0.540 \\
\hline Difficulty breathing & 0.4 & 0.2 to 0.8 & 0.020 & 0.5 & 0.3 to 0.8 & 0.009 \\
\hline Hot/feverish & 1.5 & 0.8 to 2.9 & 0.190 & 1.7 & 1.0 to 2.8 & 0.060 \\
\hline Rash & 0.8 & 0.3 to 2.1 & 0.710 & 0.8 & 0.4 to 1.8 & 0.640 \\
\hline Irritable/grouchy & 1.1 & 0.6 to 2.3 & 0.710 & 1.1 & 0.6 to 2.0 & 0.680 \\
\hline Clinginess/needing extra care & 0.8 & 0.4 to 1.8 & 0.570 & 0.8 & 0.4 to 1.5 & 0.490 \\
\hline $\begin{array}{l}\text { Low energy/tired or lost interest } \\
\text { in playing }\end{array}$ & 0.5 & 0.3 to 1.1 & 0.080 & 0.6 & 0.3 to 1.0 & 0.050 \\
\hline Poorer sleep & 2.7 & 1.5 to 5.0 & 0.001 & 2.1 & 1.3 to 3.5 & 0.004 \\
\hline Poorer feeding/poorer appetite & 1.9 & 1.0 to 3.6 & 0.060 & 1.2 & 0.7 to 2.0 & 0.570 \\
\hline Diarrhoea & 0.7 & 0.4 to 1.4 & 0.330 & 0.7 & 0.4 to 1.2 & 0.220 \\
\hline Abdominal pain & 0.8 & 0.4 to 1.5 & 0.440 & 0.8 & 0.5 to 1.5 & 0.560 \\
\hline Smelly urine & 1.0 & 0.5 to 2.0 & 0.990 & 0.8 & 0.5 to 1.6 & 0.600 \\
\hline Dark or cloudy urine & 1.9 & 0.8 to 4.2 & 0.130 & 1.6 & 0.8 to 3.3 & 0.170 \\
\hline Past history of eczema & 1.4 & 0.7 to 2.6 & 0.350 & 1.5 & 0.9 to 2.5 & 0.170 \\
\hline \multicolumn{7}{|l|}{ Examination findings } \\
\hline Abnormal ear examination & 6.5 & 2.5 to 17.2 & $<0.001$ & 5.9 & 2.7 to 12.8 & $<0.001$ \\
\hline Abnormal throat examination & 2.2 & 1.1 to 4.5 & 0.030 & 2.3 & 1.2 to 4.2 & 0.009 \\
\hline Abnormal chest examination & 13.6 & 5.8 to 32.2 & $<0.001$ & 13.4 & 6.5 to 27.7 & $<0.001$ \\
\hline Rash (on examination) & 1.6 & 0.6 to 4.4 & 0.390 & 2.2 & 1.0 to 5.0 & 0.060 \\
\hline \multicolumn{7}{|l|}{ GP working diagnosis (URTI reference) } \\
\hline LRTI & 9.5 & 3.7 to 24.5 & $<0.001$ & 6.7 & 3.0 to 14.8 & $<0.001$ \\
\hline Tonsillitis/sore throat & 119.3 & 28.2 to 504.6 & $<0.001$ & 109.5 & 30.5 to 392.8 & $<0.001$ \\
\hline Ear infection & 26.5 & 7.4 to 95.7 & $<0.001$ & 21.6 & 7.3 to 63.7 & $<0.001$ \\
\hline UTI & 12.7 & 4.4 to 36.5 & $<0.001$ & 13.1 & 5.5 to 31.0 & $<0.001$ \\
\hline Viral illness & 0.5 & 0.2 to 1.5 & 0.210 & 0.4 & 0.2 to 1.0 & 0.060 \\
\hline Other & 2.1 & 0.8 to 5.4 & 0.120 & 1.9 & 0.9 to 4.1 & 0.110 \\
\hline No GP diagnosis & 1.5 & 0.2 to 11.4 & 0.690 & 1.2 & 0.4 to 3.9 & 0.810 \\
\hline \multicolumn{7}{|c|}{ Parent/carer impression scale (normal/not unwell reference) } \\
\hline Slightly unwell & 0.9 & 0.3 to 3.1 & 0.870 & 0.6 & 0.2 to 1.6 & 0.320 \\
\hline Moderately, very, or severely unwell & 1.2 & 0.3 to 4.6 & 0.760 & 1.0 & 0.3 to 2.7 & 0.960 \\
\hline \multicolumn{7}{|c|}{ Clinician impression scale (normal/not unwell reference) } \\
\hline Slightly unwell & 2.0 & 0.9 to 4.7 & 0.110 & 2.1 & 1.0 to 4.3 & 0.050 \\
\hline Moderately, very, or severely unwell & 4.0 & 1.4 to 11.4 & 0.009 & 4.6 & 1.9 to 11.0 & 0.001 \\
\hline Temperature on examination & 0.8 & 0.6 to 1.2 & 0.300 & 0.8 & 0.6 to 1.2 & 0.260 \\
\hline \multicolumn{7}{|l|}{ Non-clinical factors } \\
\hline Age, years & 1.3 & 1.1 to 1.7 & 0.010 & 1.3 & 1.0 to 1.5 & 0.010 \\
\hline Sex (boy) (girl reference) & 1.0 & 0.6 to 1.7 & 0.900 & 0.9 & 0.6 to 1.4 & 0.590 \\
\hline
\end{tabular}

$P$-values $<0.05$ in bold. $L R T I=$ lower respiratory tract infection. URTI = upper respiratory tract infection. UTI = urinary tract infection.

working diagnosis of LRTI IOR 9.5; 95\% $\mathrm{Cl}=3.7$ to 24.5$)$, tonsillitis/sore throat $(\mathrm{OR}$

119.3: $95 \% \mathrm{Cl}=28.2$ to 504.6 ), ear infection IOR 26.5; $95 \% \mathrm{Cl}=7.4$ to 95.7 ), or UTI
IOR 12.7; $95 \% \mathrm{Cl}=4.4$ to 36.5). Children categorised as moderately, very, or severely unwell by the clinician were more likely to be prescribed antibiotics $10 \mathrm{R} 4.0 ; 95 \% \mathrm{Cl}=1.4$ 


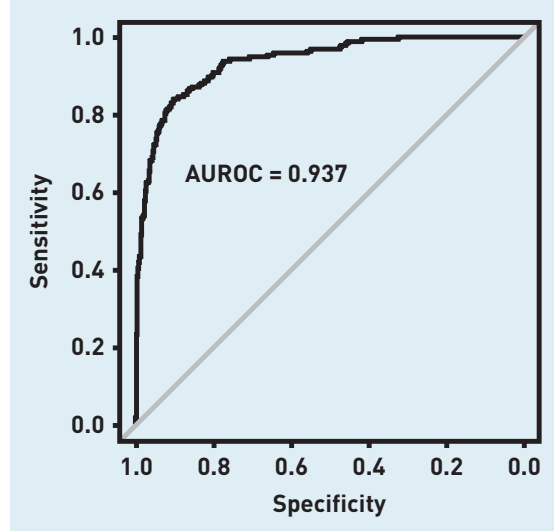

Figure 2. Predictive value of the multilevel multivariable logistic regression model for antibiotic prescribing. to 11.4) compared with children reported as normal/not unwell.

The area under the receiver operating characteristic curve (AUROC) was calculated to be 0.937 (Figure 2). This suggests that the predictive strength of the model is excellent.

Further crude (unadjusted) analyses were performed to explore the association between diagnosis and examination findings. Table 4 shows the association between GP diagnosis and examination findings for tonsillitis/sore throat, ear infection, and LRTI. A diagnosis of tonsillitis/throat infection/ sore throat was never given if the throat examination was normal or not examined. However, an abnormal throat examination did not necessarily lead to a diagnosis of tonsillitis/throat infection/sore throat, with $144(71.3 \%)$ of those with abnormal throat examination not being given a diagnosis of tonsillitis/throat infection/sore throat. A similar picture was seen with ear infections, with only two children (0.2\%) diagnosed with an ear infection in the context of a normal ear examination and 57 (53.8\%) not diagnosed with ear infection despite abnormal examination findings. LRTI was diagnosed despite no abnormal chest findings in 17 children (1.9\%), and 50 (40.7\%) of those with abnormal chest examination were not diagnosed with LRTI.

\section{DISCUSSION}

\section{Summary}

This was a prospective cohort study of 999 consecutively recruited children aged $<5$ years, presenting to general practices in Wales with an unselected acute illness. Half of all children were diagnosed with some form of RTI, and RTIs were responsible

\section{Table 4. Association between examination findings and GP diagnosis for throat, ear, and chest infections}

\begin{tabular}{|c|c|c|c|}
\hline Examination results & Diagnosed, $n(\%)$ & Not diagnosed, $n(\%)$ & Total \\
\hline \multicolumn{4}{|l|}{ Ear examination } \\
\hline Abnormal & $49(46.2)$ & 57 (53.8) & 106 \\
\hline Normal or not examined & $2(0.2)$ & 891 (99.8) & $\begin{array}{c}893 \\
P<0.001\end{array}$ \\
\hline \multicolumn{4}{|c|}{ Sore throat/tonsillitis examination } \\
\hline Abnormal & $58(28.7)$ & 144 (71.3) & 202 \\
\hline \multirow[t]{2}{*}{ Normal or not examined } & 0 & 797 (100) & 797 \\
\hline & & & $P<0.001$ \\
\hline \multicolumn{4}{|l|}{ Chest examination/LRTI } \\
\hline Abnormal & 73 (59.3) & $50(40.7)$ & 123 \\
\hline \multirow[t]{2}{*}{ Normal or not examined } & $17(1.9)$ & 859 (98.1) & 876 \\
\hline & & & $P<0.001$ \\
\hline
\end{tabular}

for $77.4 \%$ of antibiotic prescriptions. Oral antibiotics were prescribed for 261 children (26.1\%). The strongest predictors of antibiotic prescribing were a clinician diagnosis of tonsillitis/sore throat, ear infection, LRTI, or UTI and abnormal findings on chest, ear, or throat examinations. Poor sleep, increasing age, and clinician impression that the child was moderately to severely unwell also increased the likelihood of children being prescribed antibiotics. Neither fever nor parental impression of illness severity was predictive of antibiotic prescribing in the multivariable model. For tonsillitis/sore throat and ear infections, the clinician's diagnosis was a more important predictor of antibiotic prescribing than abnormal findings on examination. Diagnoses of tonsillitis/sore throat, ear infection or chest infection were rarely made if the examination findings were normal. However, abnormal examination findings did not necessarily result in the associated specific diagnoses.

\section{Strengths and limitations}

This study reports recent consultation and antibiotic prescribing for different illnesses in young children in UK general practice. In contrast to routinely collected data, the prospective design of this study allowed the exploration of the relative importance of symptoms, signs, and clinician diagnosis in predicting antibiotic prescribing. Multilevel modelling corrected for the two-level sampling process (practices and patients). Multiple imputation was used to assess the impact of missing data. This sensitivity analysis was only very slightly different from the complete case analysis. The AUROC suggested that the multilevel logistic regression model was an excellent fit.

Not all eligible children who consulted were recruited into the study. Recruitment logs demonstrated that recruited children were older than those who were not recruited. A working diagnosis of UTI, and antibiotic prescribing for suspected UTI, may have been artificially high in this study as the cohort was originally recruited to determine the prevalence of UTI. Participating GPs were aware of this, which may have altered their level of UTI suspicion or prescribing for these children. Some data were incomplete (for example respiratory ratel which may have introduced bias if they were more or less often measured in children with more severe illness or in those who were prescribed antibiotics. Pulse and respiratory rate were only recorded in about half of all children despite this being specifically requested for the study. Recording of vital signs in routine clinical practice is likely to be even lower 
than this. The confidence intervals were wide for some of the variables in the model, for example tonsillitis under GP diagnosis. This is driven by the small numbers of children with this diagnosis who were not prescribed antibiotics (Table 1). Participants' postcodes were not collected, so Townsend (deprivation) scores were based on GP practice postcodes. Although two-level sampling was corrected for using a multilevel regression model, the data were not available to explore variation by individual clinician/prescriber.

\section{Comparison with existing literature}

RTIs were diagnosed in $49.6 \%$ of consultations for acute illness. Comparisons with other literature are difficult because of differences in definitions of RTI, use of routinely collected data, and of different populations being studied. Many prospective studies have only included children with RTIs, therefore the frequency of these conditions cannot be calculated. A UK primary care study of children aged $<14$ years found that 302/407 children with a febrile illness (74\%) were recorded as having RTI. ${ }^{38}$ This is higher than in the current study but excluded children without fever. Another reason for the difference may be because 'viral illness' was not included in the current study's definition of RTI. It is likely that most 'viral illness' episodes could also have been categorised as RTIs. If these had been included, the proportion of consultations because of RTIs would be 641/999 (64.2\%). A study of 284 children laged $<14$ years) in Italy found that RTIs were diagnosed in $69.6 \%$ of consultations if flu-like illnesses were included. ${ }^{39}$

Overall, $26.1 \%$ of children in the current study received antibiotics. This is similar to an Italian study of 284 children, in which 25\% were prescribed antibiotics. ${ }^{39}$ A Dutch study of febrile children ( $<6$ years) in out-of-hours primary care found that only $12 \%$ received antibiotics. ${ }^{16} \mathrm{~A} \cup \mathrm{S}$ study found that antibiotic prescribing varied from $18 \%$ to $36 \%$ in paediatric acute visits. ${ }^{40}$ Most of the studies discussed above present data collected prior to the data collection in the current study (2010). 16,38,40 One study presents more recent data from 2011-2012, which found a similar level of antibiotic prescribing among children as in the current study. ${ }^{39}$

Children with abnormal ear, throat, and chest examination findings had an increased likelihood of receiving an antibiotic. However, a clinician diagnosis of sore throat/tonsillitis or ear infection predicted antibiotic prescribing to a greater extent than recorded examination findings. Antibiotics were prescribed for $93.1 \%$ of the children diagnosed with tonsillitis/sore throat, $82.4 \%$ with ear infection, and $70.0 \%$ with LRTI. Not all children with abnormal throat or ear examinations were diagnosed with tonsillitis/sore throat or ear infection, respectively. This suggests that clinicians are assessing features other than these specific examination findings when making their diagnosis. If there were abnormal ear examination findings and the clinician made a diagnosis of ear infection, then antibiotics were less likely to be prescribed if the child was complaining of earache. This may be due to the clinician assessing other features, perhaps more likely to prescribe antibiotics to a child with systemic signs of illness rather than local signs of ear infection. The high level of association between antibiotic prescribing and clinician diagnosis may indicate that clinicians only use certain diagnostic labels if they are planning to prescribe antibiotics. For example, a child with a sore throat may be labelled as having tonsillitis if an antibiotic is felt to be necessary, but as an URTI if an antibiotic is not felt to be necessary. Other studies have found an association between diagnostic labels and antibiotic prescribing. ${ }^{5,41,42}$

\section{Implications for research and practice}

There are still high levels of antibiotic prescribing for RTIs in children. Antibiotic prescribing was most strongly predicted by the specific diagnostic labels of ear infection and tonsillitis/sore throat. LRTI and UTI were also strong predictors of antibiotic prescribing. In some ill children, antibiotics are warranted. Further research and education is still needed to help clinicians identify those children who need antibiotics and those children in whom antibiotic prescribing can be safely reduced. The current study suggests that targeting tonsillitis/sore throat and ear infections may be a good approach. A change in emphasis away from a diagnosis-led approach Iwhere specific diagnoses have traditionally been associated with needing antibiotics) may be helpful, perhaps towards clinical scoring systems or delayed prescribing approaches. Although prescribing for URTI was low (14.5\%), antibiotics for this diagnosis represented $17 \%$ of all antibiotic prescriptions, suggesting that further improvements could also still be made in this common condition.

\section{Discuss this article}

Contribute and read comments about this article: bjgp.org/letters 


\section{REFERENCES}

1. Department of Health. UK five year antimicrobial resistance strategy 2013 to 2018. London: The Stationery Office, 2013

2. World Health Organization. Antimicrobial resistance: global report on surveillance. 2014. http://www.who.int/drugresistance/documents/ surveillancereport/en/ (accessed 26 Jun 2015).

3. Costelloe $\mathrm{C}$, Metcalfe $\mathrm{C}$, Lovering $\mathrm{A}$, et al. Effect of antibiotic prescribing in primary care on antimicrobial resistance in individual patients: systematic review and meta-analysis. BMJ 2010; 340: c2096.

4. Duffy MA, Hernandez-Santiago V, Orange G, et al. Trimethoprim prescription and subsequent resistance in childhood urinary infection: multilevel modelling analysis. Br J Gen Pract 2013; DOI: 10.3399/bjgp13X665198.

5. Goossens H, Ferech M, Vander Stichele R, et al. Outpatient antibiotic use in Europe and association with resistance: a cross-national database study. Lancet 2005; 365: 579-587.

6. Magee JT, Pritchard EL, Fitzgerald KA, et al. Antibiotic prescribing and antibiotic resistance in community practice: retrospective study, 1996-8. BMJ 1999; 319: 1239-1240.

7. Priest $P$, Yudkin P, McNulty C, Mant D. Antibacterial prescribing and antibacterial resistance in English general practice: cross sectional study. BMJ 2001; 323: 1037-1041.

8. Clavenna A, Bonati M. Adverse drug reactions in childhood: a review of prospective studies and safety alerts. Arch Dis Child 2009; 94: 724-728.

9. Little P, Gould C, Williamson I, et al. Reattendance and complications in a randomised trial of prescribing strategies for sore throat: the medicalising effect of prescribing antibiotics. BMJ 1997; 315: 350-352.

10. Standing Medical Advisory Committee. Sub-Group on Antimicrobial Resistance. The path of least resistance. London: The Stationery Office, 1998.

11. Majeed A, Moser K. Age- and sex-specific antibiotic prescribing patterns in general practice in England and Wales in 1996. Br J Gen Pract 1999; 49: 735-736.

12. Schneider-Lindner V, Quach C, Hanley JA, Suissa S. Secular trends o antibacterial prescribing in UK paediatric primary care. J Antimicrob Chemother 2011; 66: 424-433

13. Cadieux G, Tamblyn R, Dauphinee D, Libman M. Predictors of inappropriate antibiotic prescribing among primary care physicians. CMAJ 2007; 177: 877-883.

14. Cosby JL, Francis NA, Butler CC. The role of evidence in the decline of antibiotic use for common respiratory infections in primary care. Lancet Infect Dis 2007; 7(11): 749-756.

15. Cowey JR, Johnson BF, Elliott $\mathrm{V}$, et al. An association between socioeconomic deprivation and primary care antibiotic prescribing in Scotland. J Antimicrob Chemother 2014; 69: 835-841.

16. Elshout G, Kool M, Van der Wouden JC, et al. Antibiotic prescription in febrile children: a cohort study during out-of-hours primary care. J Am Board Fam Med 2012; 25: 810-818.

17. McNulty CA, Nichols T, French DP, et al. Expectations for consultations and antibiotics for respiratory tract infection in primary care: the RTI clinical iceberg. Br J Gen Pract 2013; DOI: 10.3399/bjgp13X669149

18. Ryborg CT, Søndergaard J, Lous J, et al. Factors associated with antibiotic prescribing in children with otitis media. ISRN Family Med 2013; 2013: 587452

19. Teixeira Rodrigues A, Roque F, Falcão A, et al. Understanding physician antibiotic prescribing behaviour: a systematic review of qualitative studies. Int J Antimicrob Agents 2013; 41(3): 203-212.

20. van den Broek d'Obrenan J, Verheii TJ, Numans ME, van der Velden AW. Antibiotic use in Dutch primary care: relation between diagnosis, consultation and treatment. J Antimicrob Chemother 2014; 69: 1701-1707.

21. Vodicka TA, Thompson M, Lucas P, et al. Reducing antibiotic prescribing for children with respiratory tract infections in primary care: a systematic review. $\mathrm{Br}$ J Gen Pract 2013; DOI: 10.3399/bjgp13X669167.
22. National Institute for Health and Care Excellence. Respiratory tract infections - antibiotic prescribing: Prescribing of antibiotics for self-limiting respiratory infections in adults and children in primary care. Nice guidelines CG69. London: NICE, 2008.

23. Arroll B. Antibiotics for upper respiratory tract infections: an overview of Cochrane reviews. Respir Med 2005; 99(3): 255-261.

24. Thompson PL, Spyridis N, Sharland M, et al. Changes in clinical indications for community antibiotic prescribing for children in the UK from 1996 to 2006: will the new NICE prescribing guidance on upper respiratory tract infections just be ignored? Arch Dis Child 2009; 94(5): 337-340.

25. de Bont EG, van Loo IH, Dukers-Muijrers NH, et al. Oral and topical antibiotic prescriptions for children in general practice. Arch Dis Child 2013; 98(3): 228-231.

26. Mangione-Smith R, McGlynn EA, Elliott MN, et al. Parent expectations for antibiotics, physician-parent communication, and satisfaction. Arch Pediatr Adolesc Med 2001; 155: 800-806.

27. Crocker JC, Powell CV, Evans MR, et al. Paediatric pneumonia or empyema and prior antibiotic use in primary care: a case-control study. J Antimicrob Chemother 2012; 67: 478-487.

28. Fossum GH, Lindbæk M, Gjelstad S, et al. Are children carrying the burden of broad-spectrum antibiotics in general practice? Prescription pattern for paediatric outpatients with respiratory tract infections in Norway. BMJ Open 2013; 3(1): e002285.

29. Petersen I, Hayward AC; SACAR Surveillance Subgroup. Antibacterial prescribing in primary care. J Antimicrob Chemother 2007; 60 Suppl 1: i43-i47

30. O'Brien K, Edwards A, Hood K, Butler CC. Prevalence of urinary tract infection in acutely unwell children in general practice: a prospective study with systematic urine sampling. Br J Gen Pract 2013; D0I: 10.3399/bjgp13X663127.

31. O'Brien K, Stanton N, Edwards A, et al. Prevalence of urinary tract infection (UTI) in sequential acutely unwell children presenting in primary care: exploratory study. Scand J Prim Health Care 2011; 29(1): 19-22.

32. Townsend P, Phillimore P, Beattie A. Health and deprivation: inequality and the north. London: Routledge, 1988

33. Gartner A, Lester N. Briefing paper on LSOA Townsend deprivation scores calculated from unadjusted Census data. http://www.wales.nhs.uk/sitesplus/ documents/888/TownsendBriefing.pdf (accessed 26 Jun 2015).

34. Pavia AT. Viral infections of the lower respiratory tract: old viruses, new viruses, and the role of diagnosis. Clin Infect Dis 2011; 52 Suppl 4: S284-S289.

35. National Institute for Health and Care Excellence. Feverish illness in children: assessment and initial management in children younger than 5 years. CG160. London: NICE, 2013

36. Goldstein $\mathrm{H}$, Browne W, Rasbash J. Partitioning variation in multilevel models Understanding Statistics 2002; 1(4): 223-231.

37. van Buuren S, Groothuis-Oudshoorn K. mice: Multivariate imputation by chained equations in R. J Statistical Software 2011; 45(3): 67.

38. Haj-Hassan TA, Thompson MJ, Mayon-White RT, et al. Which early 'red flag' symptoms identify children with meningococcal disease in primary care? $\mathrm{Br} J$ Gen Pract 2011; DOI: 10.3399/bjgp11X561131.

39. Giannattasio A, Lo Vecchio A, Napolitano C, et al. A prospective study on ambulatory care provided by primary care pediatricians during influenza season. Ital J Pediatr 2014: 40: 38

40. Gerber JS, Prasad PA, Localio AR, et al. Variation in antibiotic prescribing across a pediatric primary care network. J Pediatric Infect Dis Soc 2014; DOI: 10.1093/jpids/piu086

41. Deschepper R, Vander Stichele RH, Haaijer-Ruskamp FM. Cross-cultural differences in lay attitudes and utilisation of antibiotics in a Belgian and a Dutch city. Patient Educ Couns 2002; 48(2): 161-169.

42. Hutchinson JM, Jelinski S, Hefferton D, et al. Role of diagnostic labeling in antibiotic prescription. Can Fam Physician 2001; 47: 1217-1224. 